\title{
Classification of the Long QT Syndrome Based on Discriminant Analysis of T-Wave Morphology
}

\author{
JJ Struijk ${ }^{1}$, JK Kanters ${ }^{2,3,4}$, MP Andersen ${ }^{1}$, T Hardahl ${ }^{1}$, \\ C Graff ${ }^{1}$, M Christiansen ${ }^{4,5}$, E Toft ${ }^{1,6}$ \\ ${ }^{1}$ Aalborg University, Aalborg, Denmark \\ ${ }^{2}$ Copenhagen Heart Arrhythmia Research Center, Copenhagen, Denmark \\ ${ }^{3}$ Gentofte University Hospital, Copenhagen, Denmark \\ ${ }^{4}$ University of Copenhagen, Denmark \\ ${ }^{5}$ Statens Seruminstitut, Copenhagen, Denmark \\ ${ }^{6}$ Aalborg Hospital, Århus University Hospitals, Aalborg, Denmark
}

\begin{abstract}
The Long QT Syndrome (LQTS) is a genetic disorder, characterized by a long QT interval in the ECG, due to abnormal cardiac repolarization. Apart from the $Q T$ interval, various parameters, describing T-wave morphology, have been shown to have diagnostic value, but no single ECG parameter has been sufficient. In this study we present a method for discrimination among normal genotypes and mutations in the KCNQ1 (KvLQT1) and KCNH2 (HERG) genes using parameters describing T-wave morphology in terms of duration, asymmetry, flatness and amplitude. Discriminant analyses based on 3, 4, 5, or 6 parameters all resulted in perfect discrimination in a learning set of 22 subjects with respectively $3,3,2$, and 3 misclassifications in an evaluation set of 17 subjects. In a combined learning set of all 39 subjects both the 5 and 6 parameter classifiers resulted in a single misclassification.
\end{abstract}

\section{Introduction}

The congenital Long QT Syndrome (LQTS) is a disorder that typically shows a long QT interval and an abnormal T-wave morphology in the surface ECG. It is accompanied by a relatively high number of arrhythmic syncopes and sudden cardiac deaths.

The most prevalent genes affected in LQTS patients are KCNQ1 (reducing the slowly activating potassium rectifier current, $\mathrm{I}_{\mathrm{Ks}}$, leading to the disease called LQT1) and $\mathrm{KCNH} 2$ (decreasing the rapidly activating potassium rectifier current, $\mathrm{I}_{\mathrm{Kr}}$, leading to LQT2). These mutations account for more than $90 \%$ of the LQTS cases [1].

LQTS is often diagnosed after syncope, usually in childhood or as a teenager. Symptoms range from a clearly prolonged QT interval and repeated syncopes to a more or less normal ECG without further symptoms. The first symptom may, however, be sudden cardiac death.

The QT interval, the main parameter used to diagnose LQTS, is corrected for heart rate, e.g., by using Bazett's correction [2], to give QTc $\leftarrow \mathrm{QT} / \sqrt{\mathrm{RR}}$, where QT is the QT interval (s) and RR is an average RR interval (s). Using QTc as the only source of information would lead to a great number of false negatives since approximately $10 \%$ of all carriers show a normal QTc $(<440 \mathrm{~ms})$ and about $40 \%$ of the LQT2 carriers have QTc values between $410-470 \mathrm{~ms}$, which is a range that overlaps with noncarriers. Conversely, more than $10 \%$ of normals have a long QTc.

In clinical practice, QTc still is the only widely accepted quantifier of ventricular repolarization, although it is well recognized that $\mathrm{T}$-wave shape is a major qualitative source of information for diagnosis of abnormal repolarization. Thus wave shape parameters potentially provide major information to be used in the identification of LQTS. Studies have shown that LQT1 patients generally show broad $\mathrm{T}$-waves with a normal to relatively high amplitude and often without a distinct $\mathrm{T}$ onset [3]. For LQT2 patients these studies describe lowamplitude T-waves, with bifid T-waves in circa $60 \%$ of the carriers.

Recent approaches to T-wave description in the literature can be divided into three groups: 1) mathematical modelling of the T-wave [e.g., 1,4], 2) wave shape models based on the underlying physiological process [5], and 3) choice of simple parameters describing T-wave shape features, such as amplitude and duration, sometimes used in a pattern classification method [3,6]. Kanters et al. [1] obtained a perfect discrimination between LQT1 and LQT2 using a flatness parameter based on lead $V_{2}$, although there was overlap between LQT1 and Normals and a significant overlap 
between LQT2 and Normals as well. The study by Zhang et al [3] was based on 284 subjects divided into LQT1, LQT2 and LQT3: A discriminant analysis based on QTc, T-wave duration, and T-wave amplitude gave sensitivity/specificity values of $0.85 / 0.70,0.83 / 0.94$, and $0.47 / 0.63$ for the three genotypes respectively. However, the discriminant functions were based on all data available, so no evaluation was performed.

An early diagnosis of LQTS is important because proper treatment with beta-blockers or an implantable cardioverter defibrillator (ICD) can reduce the risk of sudden cardiac death. Also, it is of interest to discriminate among different LQTS forms because of cost and time reduction related to a targeted genetic screening.

In the following we present a method to discriminate among LQT1, LQT2, and normal individuals. The method is based on a Linear Discriminant Analysis using quantitative descriptors for the duration, symmetry, flatness and amplitude of the T-wave.

\section{Methods}

\subsection{Subjects}

39 subjects (19 males, 20 females) were included. The study was approved by the Ethical Committee of the counties Nordjylland and Viborg (no.: vn/2003/129). The subjects were from three groups: LQT1 $(n=12$, male/female: 5/7, mean age: $39.6 \mathrm{yrs}$, age range: 14-61 yrs), LQT2 ( $\mathrm{n}=7, \mathrm{~m} / \mathrm{f}: 4 / 3$, age:35.7 yrs, range:13-53 yrs) and Normal $(\mathrm{n}=20, \mathrm{~m} / \mathrm{f}: 10 / 10$, age:37.1 yrs, range:24-64 yrs). Genotypes were obtained for LQT1 and LQT2 patients, except for two individuals who were categorized as LQT2 by anamnesis and independent manual ECG analysis. In the Normal group there were no reports of cardiac disease or LQTS family history.

The subjects were randomly divided into a training set and an evaluation set. The training set consisted of 10 Normals, 6 LQT1, and 6 LQT2 patients. The evaluation set consisted of 10 Normals, 6 LQT1, and 1 LQT2.

\subsection{Data collection}

Standard 12-lead ECG recordings were obtained from the subjects resting in a supine position. The data were recorded with the portable CardioPerfect Resting ECG (N.V. Cardiocontrol, The Netherlands). Data were collected from 8 leads (I, II, V1-V6) with a sampling rate of $1200 \mathrm{~Hz}$. For each subject 10 seconds of ECG were used.

\subsection{Preprocessing}

All leads were baseline $(0.66 \mathrm{~Hz})$, low-pass $(50 \mathrm{~Hz})$ and notch filtered $(50 \mathrm{~Hz})$. Subsequently, in each lead fiducial points were detected, where the isoelectric line for each QT interval was established by linear interpolation of the previous PQ interval to the next PQ interval.

\subsection{Fiducial-point detection}

The following fiducial points in the ECG were detected: $\mathrm{Q}_{\text {start }}, \mathrm{R}_{\text {peak }}, \mathrm{T}_{\text {start }}, \mathrm{T}_{\text {peak }}$, and $\mathrm{T}_{\text {end }}$. The detection algorithm was based on Laguna et al. [7]: adaptive thresholding techniques applied to differentiated signals. Because of the high quality of the ECGs all QRS complexes were correctly detected. The start of the Twave, $\mathrm{T}_{\text {start }}$, was obtained with a method similar to the standard method (see [7]) of detecting $\mathrm{T}_{\text {end }}$ : the tangent at the point of the steepest slope at the leading or the trailing edge of the $\mathrm{T}$-wave crosses the isoelectric line to define $\mathrm{T}_{\text {start }}$ and $\mathrm{T}_{\text {end }}$, respectively. $\mathrm{T}_{\text {peak }}$ was defined as the maximum of the filtered $\mathrm{T}$-wave. $\mathrm{Q}_{\text {start }}$ was obtained by first detecting $\mathrm{Q}_{\text {peak }}$, then computing the highest slope before $\mathrm{Q}_{\text {peak }}$ and calculating the point where the tangent at the point with the highest slope crosses the isoelectric line.

\subsection{Quantitative T-wave parameters}

In order to quantify $\mathrm{T}$-wave morphology, multiple parameters were defined for each of four categories:

i) T-wave symmetry (6 parameters),

ii) T-wave flatness ( 8 parameters),

iii) T-wave duration (QTc plus 5 other parameters),

iv) $\mathrm{T}$-wave amplitude (7 parameters).

All parameters were calculated and averaged over all beats for each lead separately. The results from these calculations were used to calculate a standard deviation across the leads to give a measure for inter-lead variability (dispersion), which in itself may be an indicator for LQTS.

Duration, symmetry, and flatness were calculated similar to the way standard deviation, skewness and kurtosis arre calculated from a probability density function. However, the reference is not necessarily the center of the T-wave, but can also be the time of the peak.

The results from lead V5 and the standard deviations across the leads were used as the T-wave descriptors, thus giving rise to 2 times 27 parameters as candidates for descriptors of $\mathrm{T}$-wave morphology and variation of morphology across the leads.

\subsection{Discriminant Analysis}

The purpose of the statistical analysis is to develop a robust assignment rule to assign a new subject into one of 
the three groups (LQT1, LQT2 or Normal) based on a limited number of $\mathrm{T}$-wave parameters, chosen from the collection of 50. Discriminant Analysis is a standard statistical tool for such a problem [8]. A stepwise procedure based on the training set was used to choose the most significant of all 54 parameters. Four classifiers were evaluated: based on $3,4,5$, or 6 parameters, respectively.

Finally, an additional discriminant analysis was done on a set containing all subjects to see if the parameters were sufficient to classify all subjects correctly as a training set, as an indication for future development potential.

\section{Results}

Figure 1 shows that QTc is a valuable parameter for the discrimination between Normal and LQTS, but also that QTc gives no information for a separation between LQT1 and LQT2.

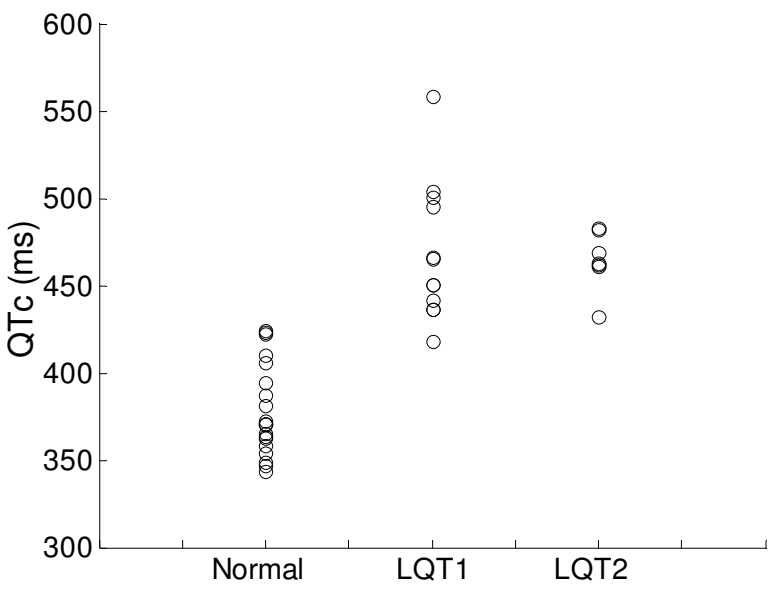

Figure 1 QTc distribution for all 39 subjects divided in the groups Normal, LQT1, and LQT2

Figure 2 shows the result of the 5-parameter classifier. The positions of all subjects are shown in a scatter plot in the two discriminant coordinates. The straight lines separating the groups in figure 2 indicate the borders between assignment areas.

The separation between the Normal groups and the LQT2 groups is very clear, as is the separation between LQT1 and LQT2. The distributions of the Normal and the LQT1 group seem to be overlapping to some extent, giving rise to a single misclassification of a Normal subject as LQT1. One LQT1 subject was misclassified as LQT2. This subject was a diabetes patient with cardioneuropathy (see discussion).

All classifiers gave a perfect classification of the training set. All classifiers also misclassified 1 Normal from the evaluation set as LQT1. For the 3, 4 and 6parameter classifier two LQT1 subjects were misclassified whereas for the 5-parameter classifier only one (the diabetes case) was misclassified.

Using all data as training data, the 3 and 4-parameter classifiers gave 2 errors, whereas the 5 and 6-parameter classifiers yielded 1 borderline misclassification (see also figures 2 and 3 ).

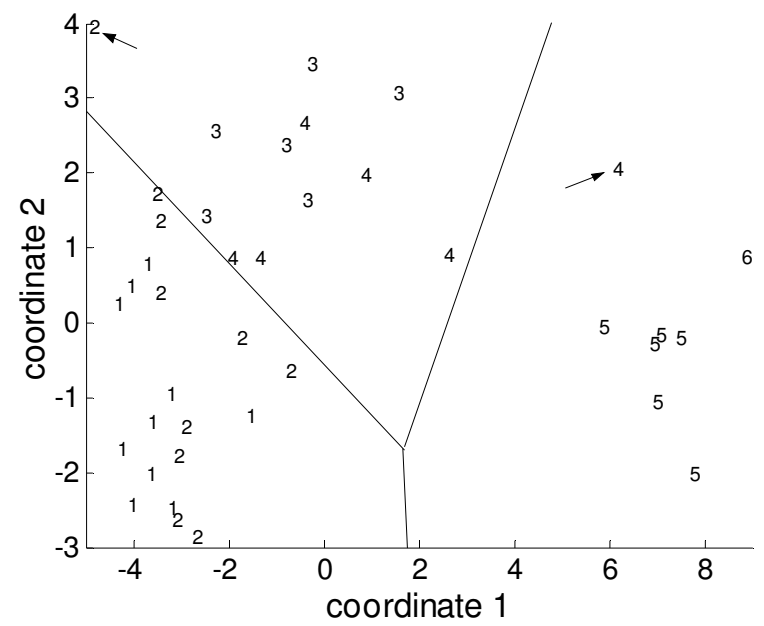

Figure 2 Result of the discriminant analysis using 5 parameters for both the training and evaluation sets: training sets have odd numbers and the evaluations sets have even numbers (1, 3, 5 and 2, 4, 6 for Normal, LQT1, LQT2 respectively). Arrows indicate misclassifications.

Table I - Stepwise entry of parameters in the Discriminant Analysis

Step Entered parameter

$1 \quad$ S2: asymmetry evaluated from $\mathrm{T}_{\text {start }}$ to $\mathrm{T}_{\text {end }}$ with $\mathrm{T}_{\text {peak }}$ as the reference

$2 \quad$ QTc

3 sdD5: standard deviation across the leads for the width of the $\mathrm{T}$-wave evaluated in the interval $\mathrm{T}_{\text {peak }} \pm 0.1\left(\mathrm{~T}_{\text {end }}-\mathrm{T}_{\text {start }}\right)$

4 sdD6: standard deviation across the leads for the width of the $\mathrm{T}$-wave evaluated in the interval $\mathrm{T}_{\text {peak }} \pm 0.1\left(\mathrm{~T}_{\text {end }}-\mathrm{T}_{\text {start }}\right)$

$5 \quad$ A7: $\mathrm{T}$ wave peak amplitude $/\left(\mathrm{T}_{\text {end }}-\mathrm{T}_{\text {start }}\right)$

6 F8: Flatness relative to $T_{\text {peak }}$ normalized by peak of $\mathrm{R}$ wave

\section{Discussion and conclusions}

The Long QT Syndrome is characterized by a 
prolonged QTc in the surface ECG. Our own dataset, and the literature as well, indicate that QTc alone often is insufficient to distinguish between normals and LQTS subjects. The need for additional measures is, therefore, obvious. Moreover, with QTc alone it would be impossible to distinguish among different forms of LQTS, which requires other classification methods than based on QTc alone.

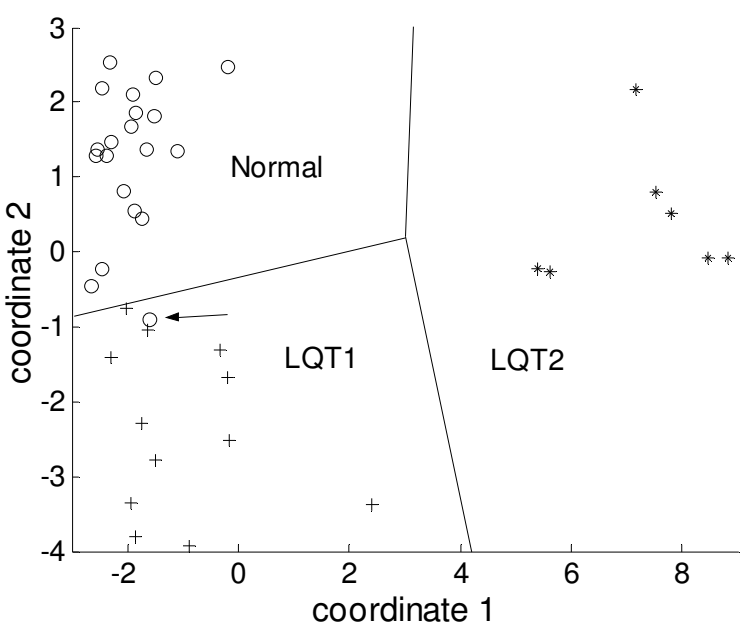

Figure 3 Result of the discriminant analysis using 5 parameters for all data. The arrow indicates the single misclassification.

The analysis shows an excellent separation between the Normal group and the LQT2 group, whereas the separation of Normals and LQT1s seems to be more critical. The great separation between Normals and LQT2s strongly encourages the use of the parameters dominating function 1 for the detection of drug-induced LQTS, since malignant drug-induced QT prolongations have been associated with blocking of $\mathrm{I}_{\mathrm{Kr}}$ channels, i.e., the channels responsible for LQT2 [27].

It was confirmed, by the parameters entered in the discriminant functions, that QTc is one of the most important parameters, but that descriptors for $\mathrm{T}$-wave duration, flatness and symmetry are indeed necessary to obtain a correct classification.

The choice of descriptive parameters was founded on, initially, qualitative descriptions of $\mathrm{T}$-wave morphology as seen in the set of subjects and in the literature $[1,3]$. This approach quickly led to a focus on duration, amplitude, asymmetry and flatness as high-level descriptors to be translated into specific quantitative descriptors. However, because the $\mathrm{I}_{\mathrm{Ks}}$ and $\mathrm{I}_{\mathrm{Kr}}$ currents have their maxima around the early part of phase 3 of the action potential [9], it was expected that differences among the three groups might be highlighted best when paying additional attention on the time interval around the peak of the T-wave. This second approach to the problem led to the inclusion of parameters that focused on the central part of the T-wave.

Interestingly, two of the parameters entered are standard deviations across the leads, which means that dispersion-like features, pointing at changes in the $\mathrm{T}$ wave loop orientation and T-wave loop width, may be informative with relation to the classification of LQTS. A clear physiological interpretation is not readily available.

A training set of 22 subjects divided over 3 groups is very small. Efforts have to be directed, first and foremost, to increase the numbers of subjects. It is therefore, most encouraging that it is possible with 5 or 6 parameters to obtain an almost perfect classification within the combined group of 39 subjects.

\section{References}

[1] Kanters JK, Fanoe S, Larsen LA, Thomsen PEB, Toft E, Christiansen M. T-wave morphology analysis distinguishes between KvLQT1 and HERG mutations in Long QT Syndrome. Heart Rhythm 2004; 1:285-292.

[2] Bazett HC. An analysis of the time-relations of electrocardiograms. Heart 1920; 7:353-370.

[3] Zhang L, et al. Spectrum of ST-T Wave Patterns and Repolarisation Parameters in Congenital Long-QT Syndrome: ECG findings identify genotypes. Circulation 200; 102:2849-2855.

[4] Padrini R, Butrous G, Statters D, Camm AJ, Malik M. Morphological algebraic models of the TU-wave patterns in idiopathic long QT syndrome. Int J Cardiol 2001; 77:151-162.

[5] diBernardo D, Langley PH, Murray A. Effect of changes in heart rate and in action potential duration on the electrocardiogram T wave shape. Physiol Meas 2002; 23:355-364.

[6] Couderc J-PH, Zareba W, Moss A.J. Drug-induced changes of ventricular repolarization: new incentives for quantifying $\mathrm{T}$ wave morphology. Int $\mathrm{J}$ Bioelectromagnetism 2003; 5:167-170.

[7] Laguna P, Thakor NV, Caminal P, Jane R, Yoon HR, Bayes de Luna A, Marti V, Guindo J. New algorithm for QT interval analysis in 24-hour Holter ECG: performance and applications. Med \& Biol Eng \& Comput 1990; 28:67-73.

[8] Seber, GAF. Multivariate Observations. John Wiley \& Sons, New York, 1984.

[9] Zipes DP, Jalife J (eds). Cardiac Electrophysiology - From Cell to Bedside. Philadelphia, W.B. Saunders, 1995.

Address for correspondence:

Johannes Jan Struijk

Center for Sensory-Motor Interaction

Department of Health Science and Technology

Aalborg University

Fredrik Bajers Vej 7D2

DK-9220 Aalborg East

Denmark

E-mail: jjs@hst.aau.dk 